\title{
Cytology of Extrapulmonary Tuberculosis of Masseter Muscle: A Rare Case Report
}

\author{
R. Ramya*, G. Barathi and D. Prathiba
}

Department of Pathology, Sri Ramachandra University, Chennai, India

\begin{abstract}
Tuberculosis (TB) is a major health hazard Worldwide. India bears a huge burden of world's TB, according to World Health Organzation (WHO) statistics. The most common form of presentation is pulmonary tuberculosis. Extra Pulmonary presentation of TB in masseter muscle is extremely rare. We present a case of 26 year old male with right sided facial swelling, Fine Needle Aspiration Cytology (FNAC) of which revealed TB of masseter muscle. FNAC is a simple,cost effective out patient procedure that can aid in the early diagnosis of orofacial lesions such as extra pulmonary Tuberculosis. The purpose of this paper is to state the significance of early diagnosis of orofacial Tuberculosis and the crucial role played by FNAC in the diagnosis.
\end{abstract}

Keywords: Masseter muscle, Extra pulmonary, Tuberculosis, Cytology

\section{Introduction}

Tuberculosis has been the alarming World health problem for many centuries. India holds a huge burden of Worlds $\mathrm{TB}^{[1]}$.Pulmonary tuberculosis is the most common form of presentation. Extra pulmonary presentations can occur in Lymph nodes, Gastrointestinal tract, Liver, Genitourinary system, Central nervous system, Skin, Bones etc ${ }^{[2]}$. TB occurring in orofacial region is very rare ${ }^{[3]}$. Tuberculosis of the facial muscle is extremely rare, accounting to less than $1 \%$ of all TB cases ${ }^{[2]}$. The real challenge lies in the early diagnosis of such rare cases.Herein we report a case of extra pulmonary TB of masseter muscle diagnosed primarily by FNAC.The prompt onset of treatment has led to complete resolution of the disease.Hence the role of FNAC for orofacial lesions can be very vital for the early diagnosis of TB.

\section{Case Report}

A 26 year old male presented to the Pathology department with right sided face swelling of one month duration, which was associated with pain during swallowing. There was no history of fever or any other constitutional symptoms.

On examination, the swelling was cystic and dumbbell shaped, measuring $7 \times 4 \mathrm{~cm}$. It was soft in consistency, non-fluctuant, with normal overlying skin, extending from nasolabial fold to epicanthus of right eye (Figure 1). Complete Blood Count (CBC) was within normal limits. Erythrocyte Sedimentation Rate(ESR) was $15 \mathrm{~mm} / \mathrm{hr}$. Mantoux test was not performed. MRI of head and neck revealed a well circumscribed bilobed cystic lesion in the right masseter muscle extending up to right zygomatic region. There was no bony erosion noted(Figure 2). HIV status of the patient is Non reactive.
Fine Needle Aspiration of the lesion yielded $4.5 \mathrm{ml}$ of thick turbid pus. Cytology revealed epithelioid granuloma and dense inflammatory cells, composed predominantly of neutrophils and macrophages in a necrotic background(Figure 3 \& 4). Special stains for Acid Fast Bacilli (AFB) was positive. This confirmed the diagnosis of tuberculosis (Figure 5). The patient was diagnosed of Tuberculosis by a simple FNAC procedure.

The patient underwent anti tuberculous treatment for 6 months. He responded well to the treatment. His swelling subsided completely and he was disease free after 6 months. On repeated follow up, the patient was symptom free.

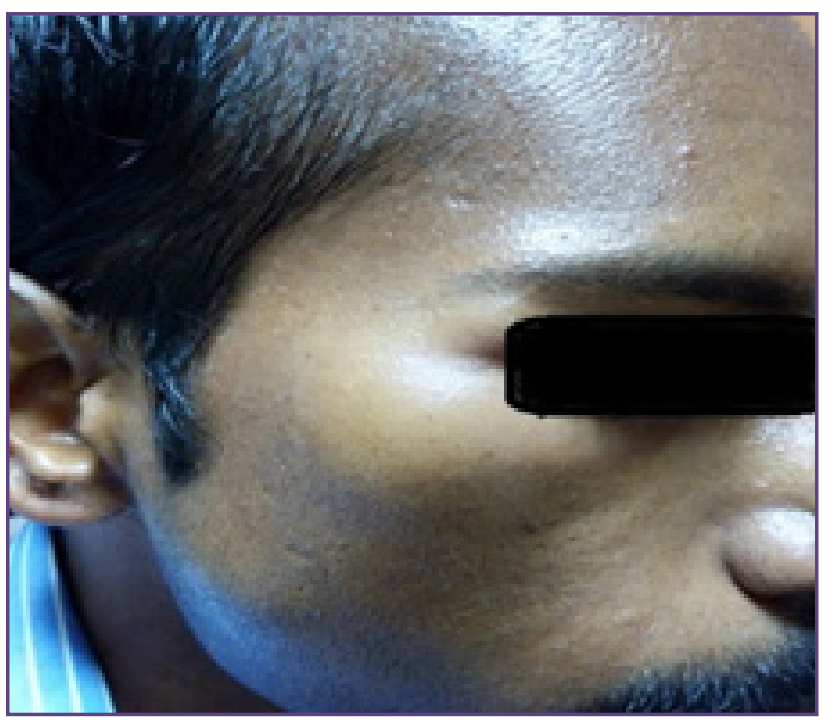

Fig.1: Patient with dumbbell shaped swelling in the right side of the face. 


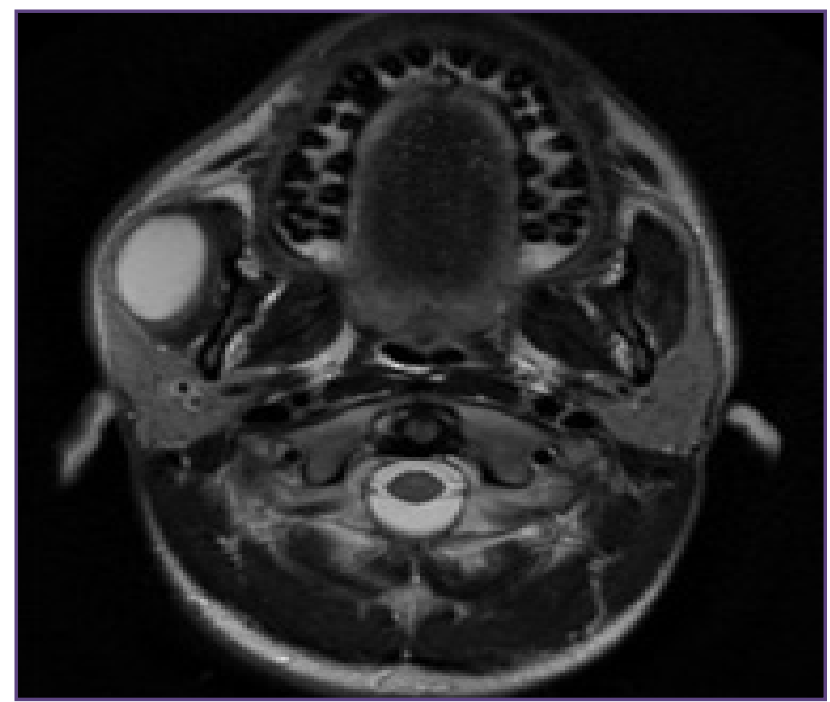

Fig. 2: MRI of head and neck showing well circumscribed cystic lesion in right side (Arrow).

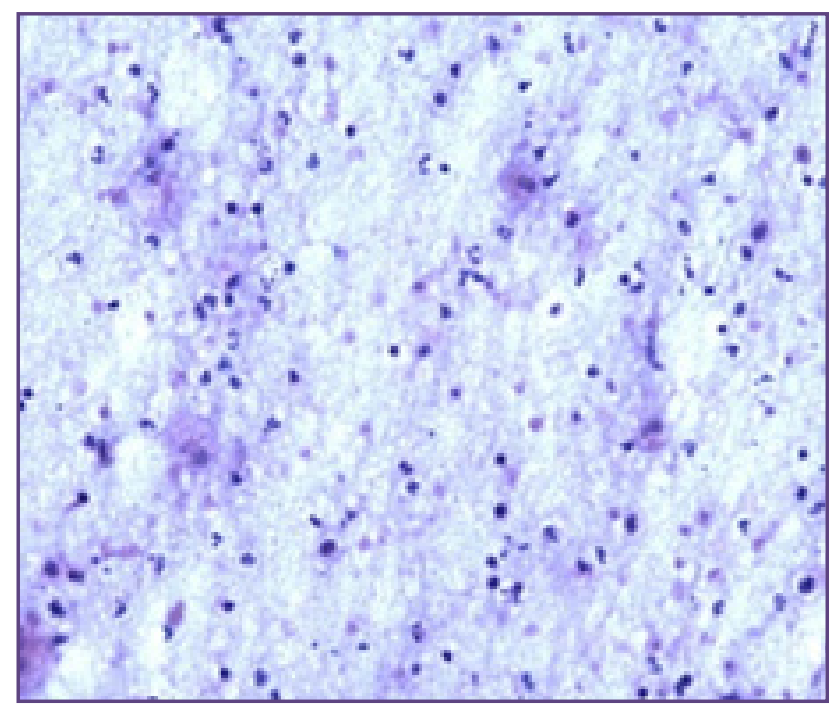

Fig. 4: FNAC of swelling-H \&E 200X showing inflammatory cells in a necrotic background.

\section{Discussion}

Tuberculosis has been haunting the mankind for a very long period. The reigns of the most cruel dictators have met its end, but Tuberculosis is a mere disease which still reigns this world from time immemorial. It has indeed evolved with a fierce rage towards mankind. TB is an alarming health hazard worldwide, with an estimated 9 million new cases and 1.5 million deaths every year ${ }^{[1]}$.

TB is a chronic infectious granulomatous disease which can involve almost any part of the body.The causative organism for this dreadful disease is Mycobacterium tuberculosis also known as Koch's bacillus. Pulmonary Tuberculosis is

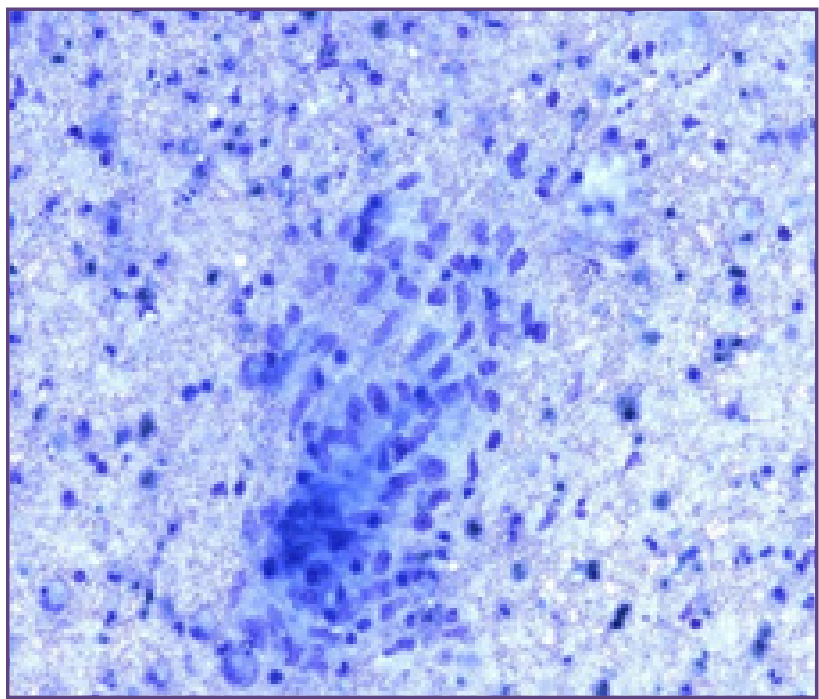

Fig. 3: FNAC of swelling-MGG 200X showing granuloma with inflammatory cells in the background.

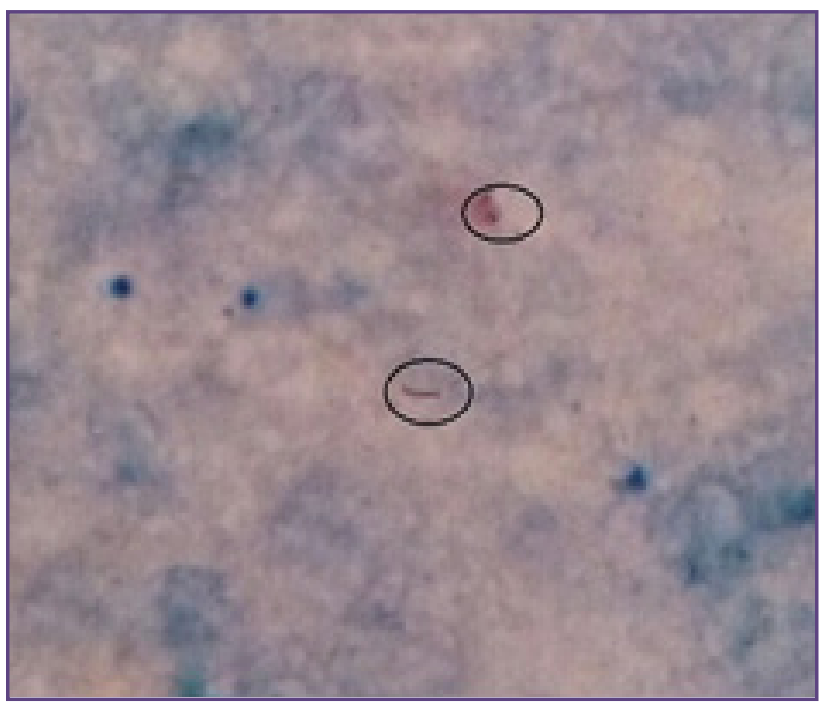

Fig. 5: AFB stain-Oil Immersion Field showing Acid Fast Bacilli (Circled).

the most common form of presentation ${ }^{[8]}$. Extra pulmonary Tuberculosis (EPTB) refers to TB involving organs other than the lungs ${ }^{[11]}$. They constitute about $15 \%$ to $20 \%$ of all TB cases affecting immunocompetent people ${ }^{[4]}$. EPTB is on the rise worldwide. EPTB is often seen in immunocompromised patients, especially associated with HIV patients ${ }^{[12]}$.

TB of head and neck region comprises about $10 \%$ of all $\mathrm{EPTB}^{[12]}$. Orofacial tuberculosis is indeed a rare form of presentation and it can occur in sites such as gingiva, tongue, fausial pillars, buccal mucosa, temperomandibular joints and muscles of mastication ${ }^{[3]}$. Tuberculous oral lesions accounts for 0.05 to $5 \%$ of all TB cases $^{[2,8]}$. 
The musculoskeletal TB accounts for nearly 3\% of all $\mathrm{EPTB}^{[7]}$. Tuberculosis in masseter muscle is an extremely rare presentation, accounting to less than $1 \%$ of EPTB occurring in orofacial region ${ }^{[3]}$. TB of masseter muscle can cause trismus or present with submasseteric abscess ${ }^{[6,7]}$. Majority of these lesions are secondary lesions occuring mainly due to reactivation of latent primary infection such as cutaneous TB like scrofuloderma and spreading to sites like masseter muscle ${ }^{[6]}$. Since there is no specific pathognomonic signs for TB in such cases, diagnosis can be very crucial $^{[3]}$. In our case, the patient had no signs to point towards TB, except for the tender swelling in his right cheek.

Orofacial lesions can occur in the form of ulcers, fissures, tuberculomas or nodules ${ }^{[5]}$. These lesions may be painful or painless, solitary or multiple, irregular, well circumscribed ulcer ${ }^{[5,9]}$.The main differential diagnosis for such lesions should be Tuberculosis. The diagnosis of these pauci bacillary lesion is often overlooked as it has variable modes of presentation ${ }^{[3,6]}$.India being one of the capitals for TB, any suspicious lesion should always be investigated for TB. FNAC of these lesions are mandatory as it can guide us towards the cause. FNAC could be crucial in the initial diagnosis of diseases like Tuberculosis. FNAC proves to be less invasive and rapid in diagnosis for such cases.

If FNAC is inconclusive due to the absence of granuloma or negative for AFB in the smear, diagnosis can be established by histopathological and microbiological study of tissue specimen ${ }^{[4]}$. Confirmation of the diagnosis can also be done by sending the material aspirated by FNAC for Polymerase Chain Reaction (PCR) analysis.

In our case, cytology showed definite granuloma, followed which Acid fast bacilli was demonstrated by AFB staining. Thus clinching the early diagnosis of Tuberculosis by mere FNAC.

\section{Conclusion}

Extra pulmonary tuberculosis detection can be very challenging to the medical world .In Tuberculosis prevalent country like India, its always better to suspect TB if there is a long standing lesion in the head and neck region. Any orofacial lesion should undergo FNAC, as it can be the initial step towards diagnosis. EPTB occurring in the orofacial region,such as masseter muscle is extremely rare. FNAC being a simple cost effective test, when done meticulously along with a thorough search for AFB on smear, can point to the definitive diagnosis. For patients who cannot afford ancillary molecular testing, FNAC \& AFB staining of smears can prove to be a boon for early diagnosis of the disease. Definitive diagnosis of such cases of TB is vital, in order to initiate timely treatment regimen and to prevent complications.

\section{Acknowledgements}

We place our sincere thanks to the patient who was utmost cooperative till date.

\section{Reference}

1. World Health Organization. Global tuberculosis report 2015. World Health Organization; 2015.

2. Jain P, Jain I. Oral manifestations of Tuberculosis: step towards early diagnosis. J Clin Diagn Res. 2014 Dec $1 ; 8(12): 18-21$.

3. Andrade NN, Mhatre TS. Orofacial tuberculosis-a 16-year experience with 46 cases. Journal of Oral and Maxillofacial Surgery. 2012 Jan 31;70(1):e12-22.

4. Kishore DN, Geetha NT, Umashankara KV, Rai KK. Submasseteric Tuberculous Lesion of Mandible: Report of a Case and Review of the Literature. Case reports in dentistry. 2014 Jun 22;2014.

5. Prabhu SR, Daftary DK, Dholakia HM. Tuberculous ulcer of the tongue: report of case. Journal of oral surgery (American Dental Association: 1965). 1978 May;36(5):384-6.

6. Mascarenhas S, Tuffin JR, Hassan I. Tuberculous submasseteric abscess: case report. British Journal of Oral and Maxillofacial Surgery. 2009 Oct 31;47(7):566-8.

7. Yadav S, Madan K. Tuberculous 'lock jaw'. BMJ case reports. 2015 Jul 9;2015:bcr2015211183.

8. Kamala R, Sinha A, Srivastava A, Srivastava S. Primary tuberculosis of the oral cavity. Indian Journal of Dental Research. 2011 Nov 1;22(6):835.

9. Kannan S, Thakkar P, Dcruz AK. Tuberculosis masquerading as oral malignancy. Indian journal of medical and paediatric oncology: official journal of Indian Society of Medical \& Paediatric Oncology. 2011 Jul;32(3):180.

10. Sharma SK, Mohan A. Tuberculosis: From an incurable scourge to a curable disease-journey over a millennium.

11. Lee JY. Diagnosis and treatment of extrapulmonary tuberculosis. Tuberculosis and respiratory diseases. 2015 Apr 1;78(2):47-55.

12. Pandurang K, Shenoy VS, Bhojwani K, Alva A, Prasad V, Gandla S. Tuberculosis in the head and neck in India: down but not yet dead. J Mycobac Dis. 2014;4(148):2161-1068.

*Corresponding author:

Dr Ramya. R, 6/4, Shreshtariverview apt, Woodcreek county, Nandampakkam, Chennai-89. India

Phone: +91 9962500343

Email: sungeeth.ramya@gmail.com

Financial or other Competing Interests: None.

Date of Submission : 07.11.2016

Date of Acceptance : 09.03.2017

Date of Publication : 29.05.2017 